\title{
CC PRE-AMP PLATFORM: STRUCTURAL ANALYSIS
}

Steven Sakla

June 9,1994

Approved:

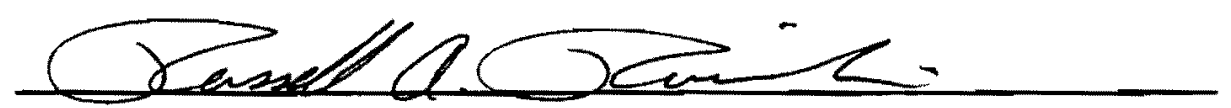

D0 Engineering Note: $3740.210-E N-406$ 


\section{INTRODUCTION}

The structure in question is an existing personnel access platform located in the D-Zero Assembly Building. This platform is used to access the preamplifiers located on the central calorimeter. The platform will need to be shortened to a "fold-out" length of $36 \mathrm{in}$. due to some modifications recently made to the detector. The purpose of this note is to determine whether this modification affects the safety and the rated load of the platform.

Three of the load carrying members were analyzed; the gusset supports, the hinge supports, and the hinge pins. The long and short beams which make up the platform's flooring were not analyzed (see pg. 1). The long beams will not be affected by this modification because there is no change in the length of their moment arms. The short beams will be affected, but will see a smaller bending stress because of the decrease in the length of their moment arms. Any weld results previously found will also not be affected.

\section{ANALYSIS}

The first member analyzed was the gusset support. This supporting member was statically indeterminate. The reaction forces as shown on $\mathrm{pg} .2$ are: $A_{x}=B_{x}=3594 \mathrm{lbs}$. and $A_{y}+B_{y}=800 \mathrm{lbs}$. The gusset was divided into three sections: $A, B$, and $C$ which were all analyzed separately. Maximum bending and transverse shear stresses were calculated for each of the three sections. Section $C$ was analyzed by defining the area, moment of inertia, and the moment as a function of $x$ since these three values changed with the position at which the member was being analyzed. The maximum bending and transverse shear stresses were calculated as $x$ varied from $x=0$ to $x=33.5$ by using a Fortran program. The maximum bending stress was $10,005 \mathrm{psi}$. and the maximum shear stress was 2403 psi. According to AISC specifications 1.5.1.4 and 1.5.1.2, respectively, the maximum allowable bending stress is equal to $0.66 \times$ (yield stress $)=23.8 \mathrm{ksi}$. and the maximum allowable shear stress is equal to $(0.40) \times($ yield stress) $=14.4 \mathrm{ksi}$.

The next member analyzed was the hinge which is welded to the gusset support. Both the upper and lower hinges were analyzed. The maximum bending stress calculated was 22,986 psi. (upper hinge), the maximum normal stress was 7663 psi. (upper hinge), and the maximum shear stress was 640 psi. 
(upper hinge). For a combined loading (compressive and bending stress), AISC specification 1.6.1 states that the sum of the ratios of the computed axial stress to 0.6 times the yield stress and the computed bending stress (in the $x$ and $y$ directions) to the allowable bending stress must be less than or equal to one. Both parts of the hinge met this criteria. The upper and lower hinges welded to the support bridge were also analyzed. The maximum normal stress for this member was $8170 \mathrm{psi}$. (upper hinge), the maximum bending stress was 8710 psi. (lower hinge), and the maximum shear stress was 2734 psi (lower hinge). Both parts of this hinge also met AISC specifications. The lower pin connecting the two parts of the hinge has a maximum shear stress of 15,504 psi which is below the maximum allowable stress of $18.7 \mathrm{ksi}$.

\section{CONCLUSION}

According to the calculations performed, this platform should still be safe after it's length is shortened to 36 in. provided the maximum load stays under 800 Ibs. It also is within the allowable limits for normal, bending, and shear stress according to standards devised by the American Institute of Steel Construction. 


\section{SUMMARY}

\begin{tabular}{|c|c|c|c|c|}
\hline MEMBER & $\begin{array}{c}\text { MAX. } \\
\text { NORMAL } \\
\text { STRESS }\end{array}$ & $\begin{array}{c}\text { MAX. } \\
\text { BENDING } \\
\text { STRESS }\end{array}$ & $\begin{array}{c}\text { MAX. SHEAR } \\
\text { STRESS }\end{array}$ & $\begin{array}{c}\text { PASS AISC } \\
\text { SPECS. }\end{array}$ \\
\hline Gusset Support & - & $10,005 \mathrm{psi}$ & $2,403 \mathrm{psi}$ & YES \\
\hline $\begin{array}{c}\text { Upper Hinge } \\
\text { Welded to } \\
\text { Gusset }\end{array}$ & $7,663 \mathrm{psi}$ & $22,986 \mathrm{psi}$ & $640 \mathrm{psi}$ & YES \\
\hline $\begin{array}{c}\text { Lower Hinge } \\
\text { Welded to } \\
\text { Gusset }\end{array}$ & $3,832 \mathrm{psi}$ & $11,497 \mathrm{psi}$ & 0 & YES \\
\hline $\begin{array}{c}\text { Upper Hinge } \\
\text { Welded to } \\
\text { Support Bridge }\end{array}$ & $8,170 \mathrm{psi}$ & $7,756 \mathrm{psi}$ & $2,733 \mathrm{psi}$ & YES \\
\hline $\begin{array}{c}\text { Lower Hinge } \\
\text { Welded to } \\
\text { Support Bridge }\end{array}$ & $7,663 \mathrm{psi}$ & $8,710 \mathrm{psi}$ & $2,734 \mathrm{psi}$ & YES \\
\hline $\begin{array}{c}\text { Upper Hinge } \\
\text { Pin }\end{array}$ & - & - & $\begin{array}{c}14,015 \mathrm{psi} \\
\text { (nominal) }\end{array}$ & YES \\
\hline $\begin{array}{c}\text { Lower Hinge } \\
\text { Pin }\end{array}$ & - & - & $\begin{array}{c}15,504 \mathrm{psi} \\
\text { (nominal) }\end{array}$ & YES \\
\hline
\end{tabular}

${ }^{*} \mathrm{~F} y=36 \mathrm{ksi}$.

\section{ALLOWABLE STRESSES:}

Normal: $F_{\mathrm{n}}=0.45 \mathrm{~F}_{\mathrm{y}}=16.2 \mathrm{ksi}$

Bending: $\mathrm{F}_{\mathrm{b}}=0.66 \mathrm{~F} \mathrm{y}=23.8 \mathrm{ksi}$.

Shear: $\mathrm{F}_{\mathrm{V}}=0.40 \mathrm{Fy}=14.4 \mathrm{ksi}$.

Shear (pin): $F_{V}=0.22(85 \mathrm{ksi}$.) $=18.7 \mathrm{ksi}$.

Combined (Shear \& Bending): $\left(\mathrm{f}_{\mathrm{a}} / 0.60 \mathrm{~F}_{\mathrm{y}}\right)+\left(\mathrm{fbx}_{\mathrm{b}} / \mathrm{Fbx}\right)+\left(\mathrm{f}_{\mathrm{by}} / \mathrm{F}\right.$ by $) \leq 1.0$

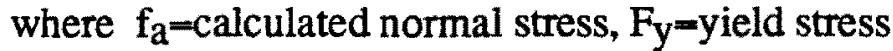

$\mathrm{f}_{\mathrm{b}}=$-calculated bending stress, $\mathrm{F}_{\mathrm{b}}=$-allowable bending stress 


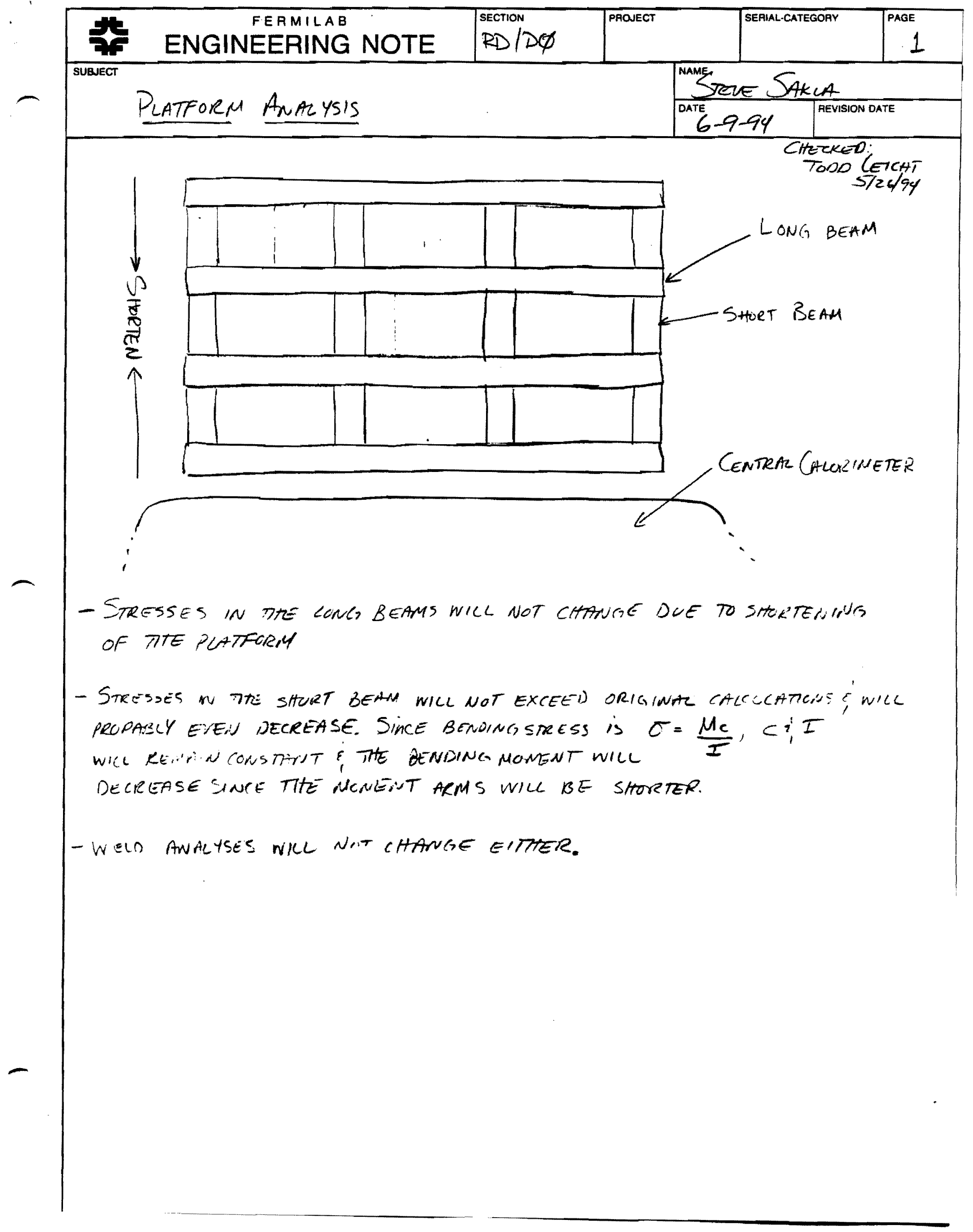




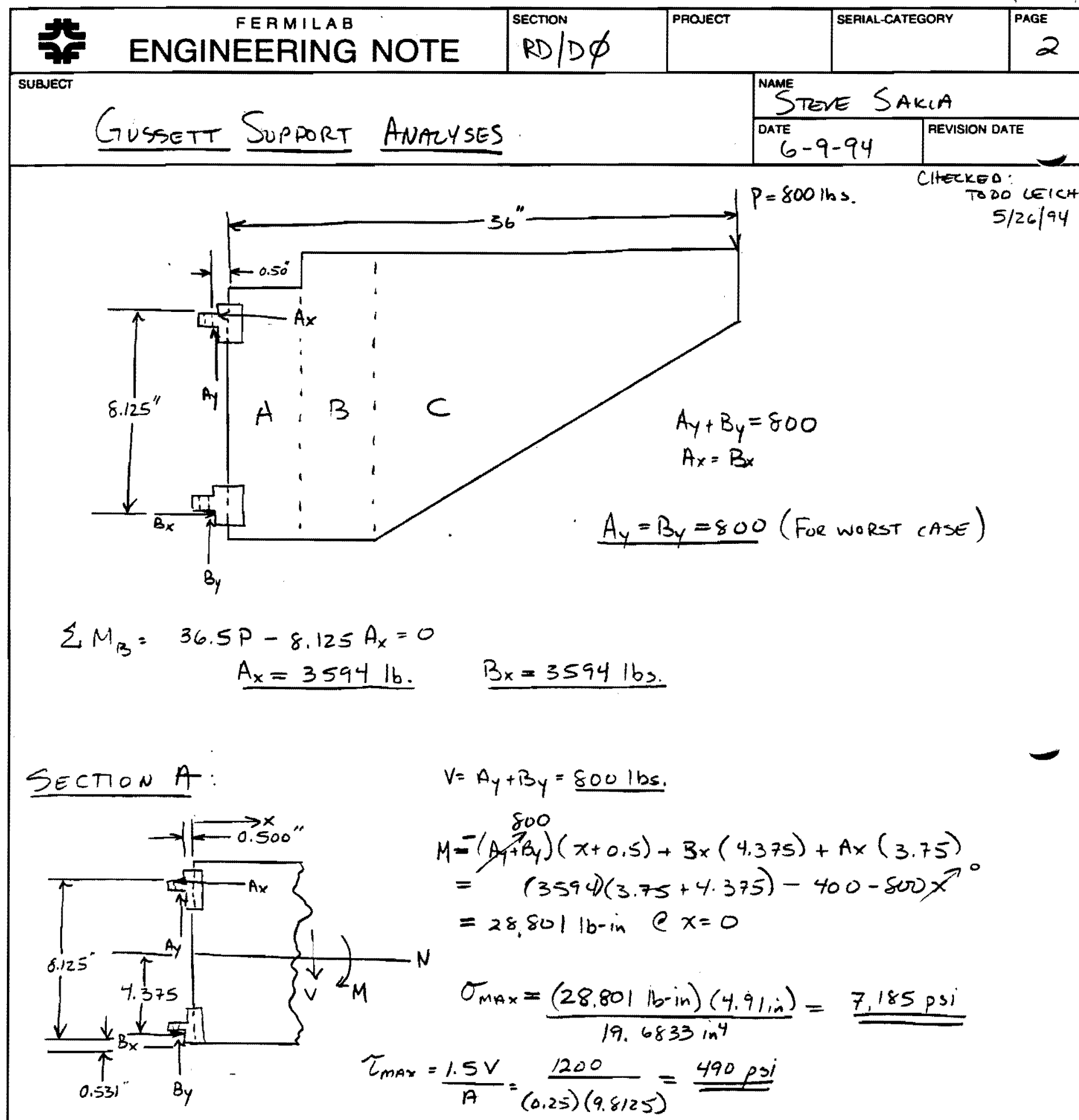




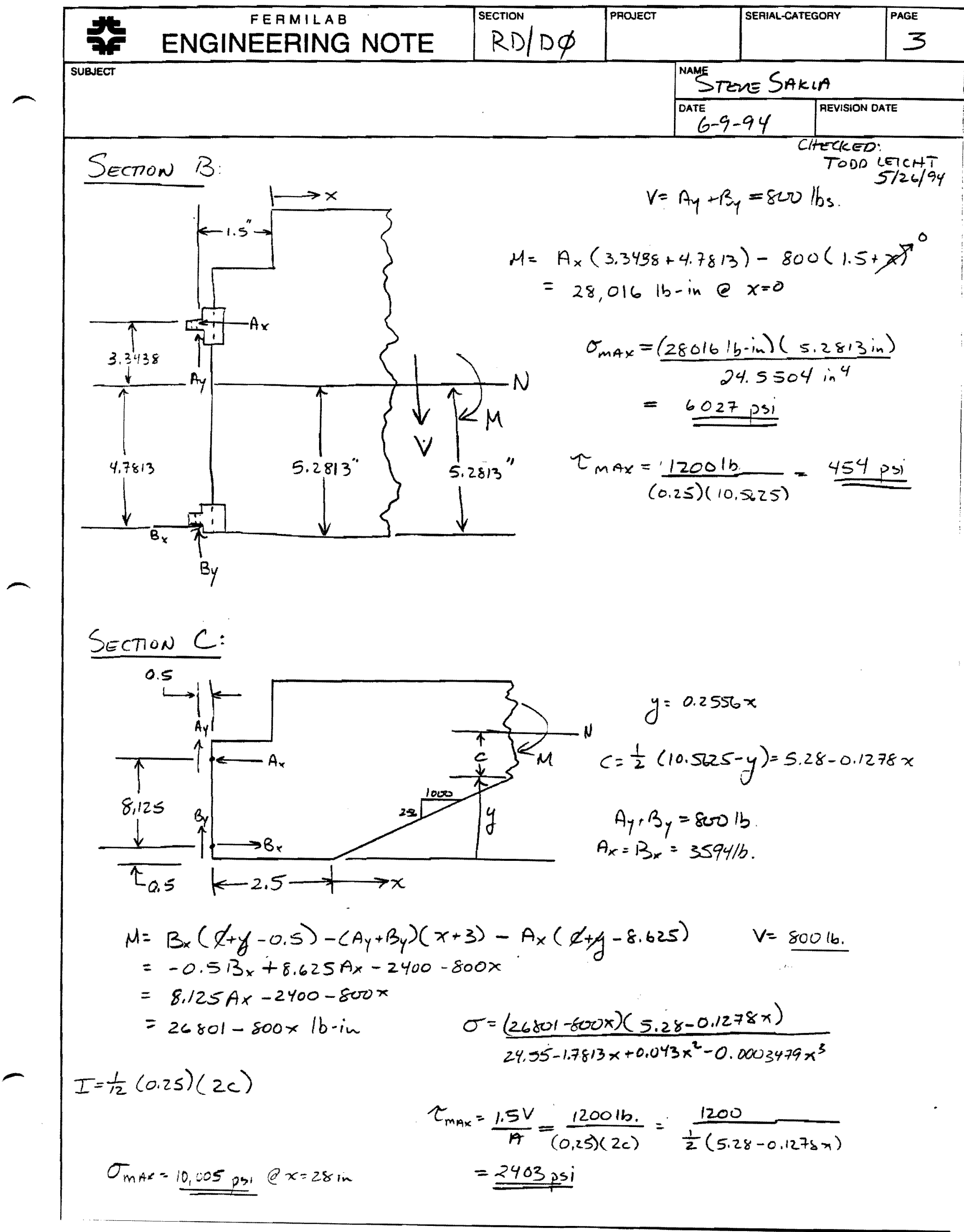




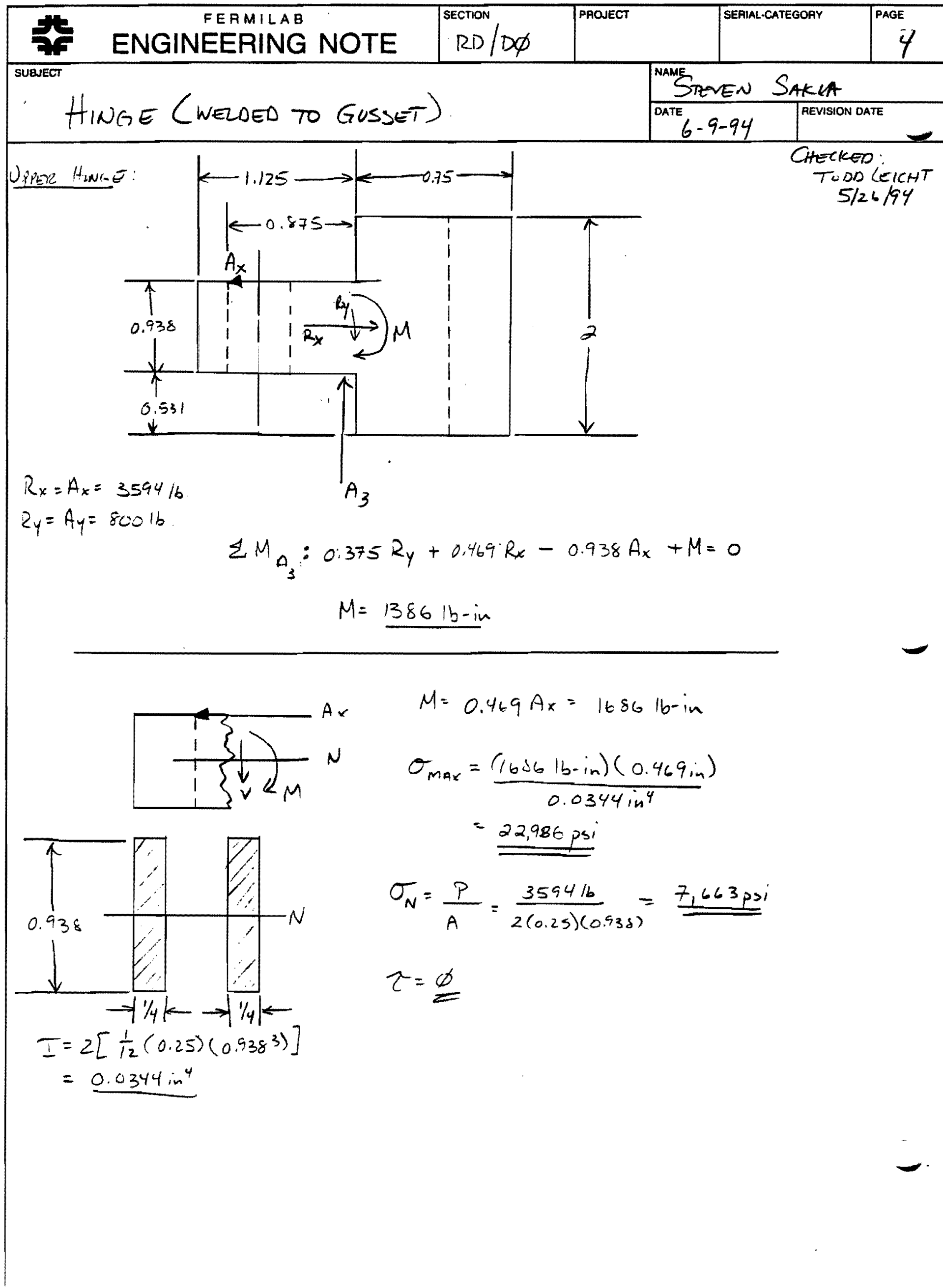




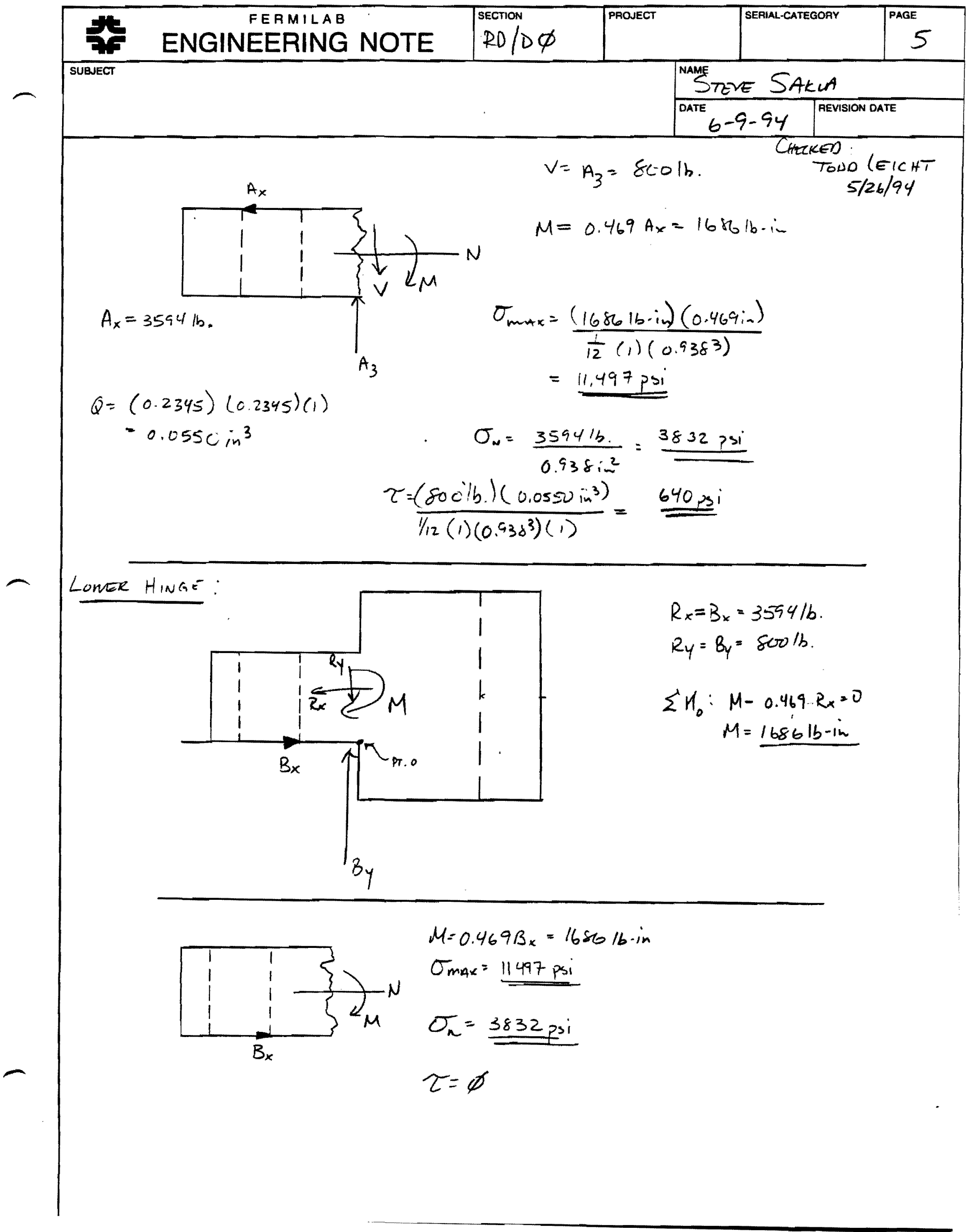




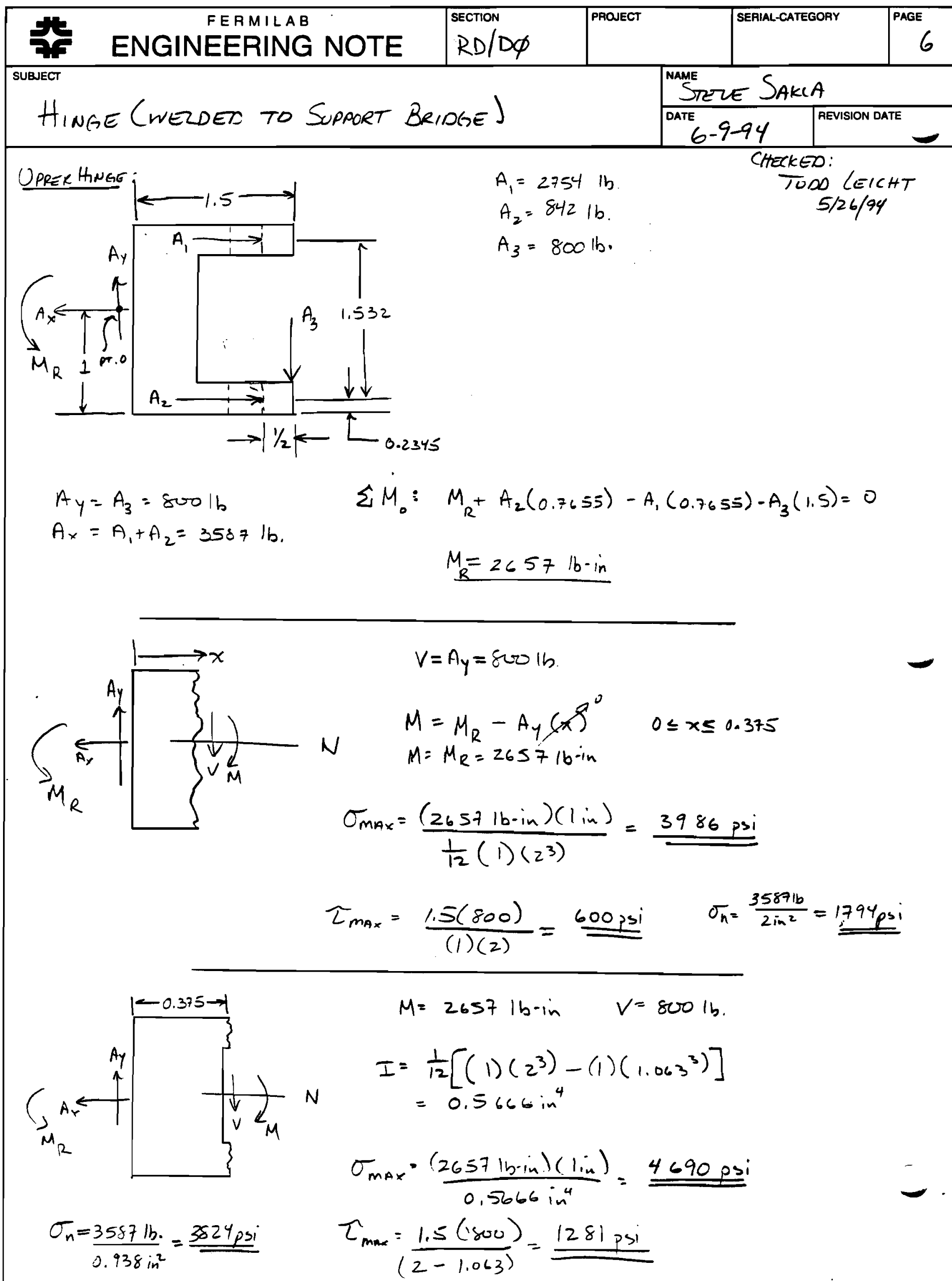




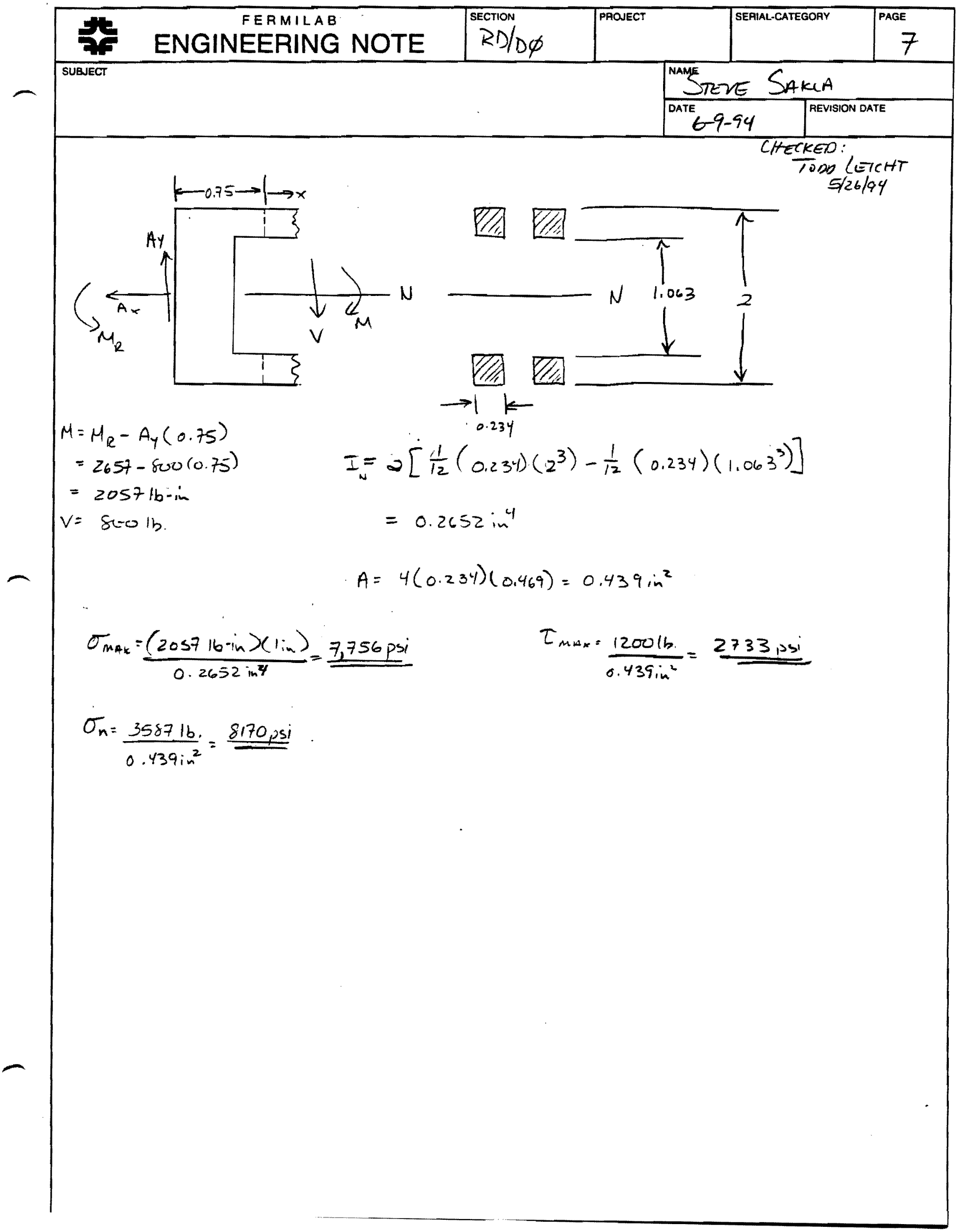




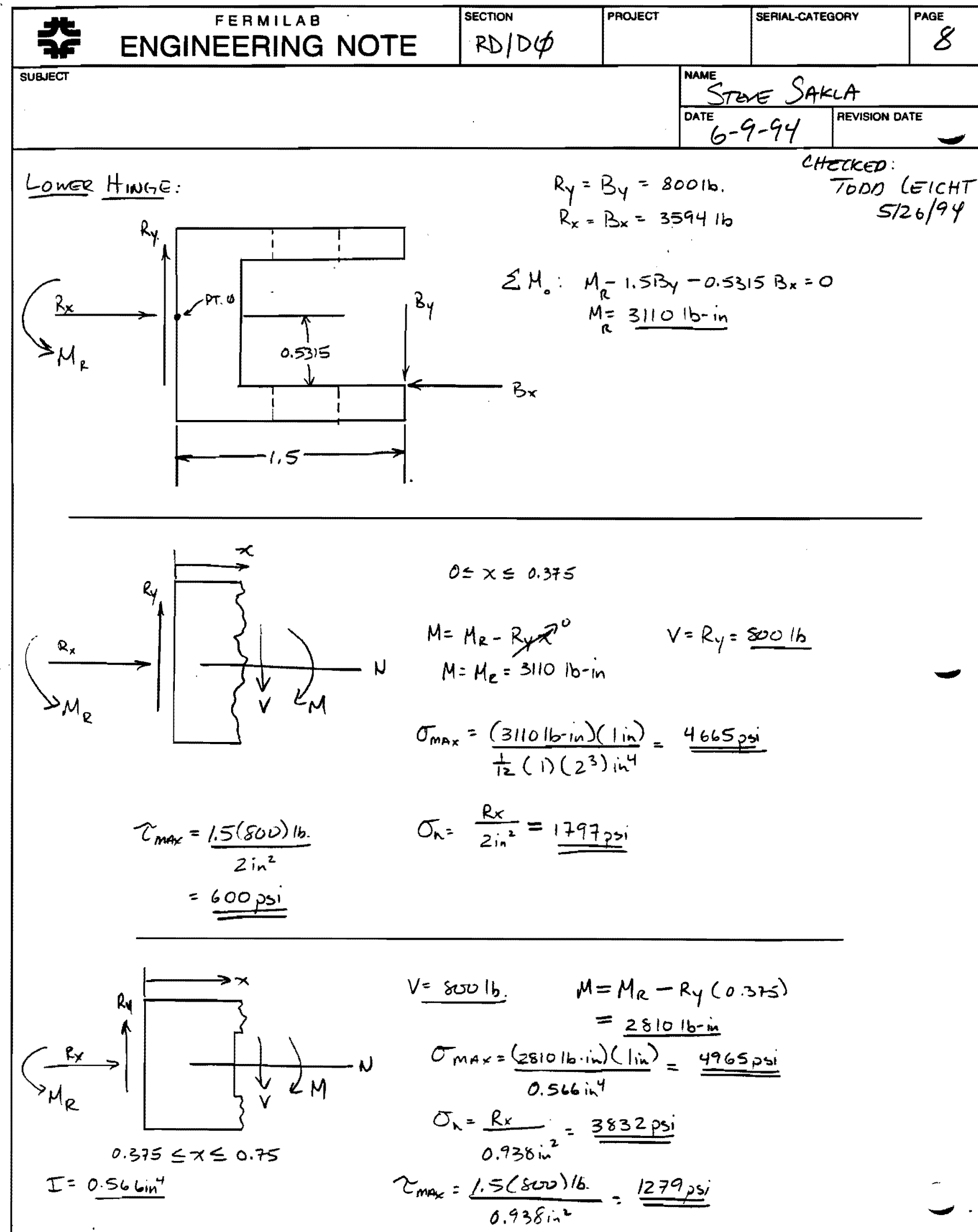




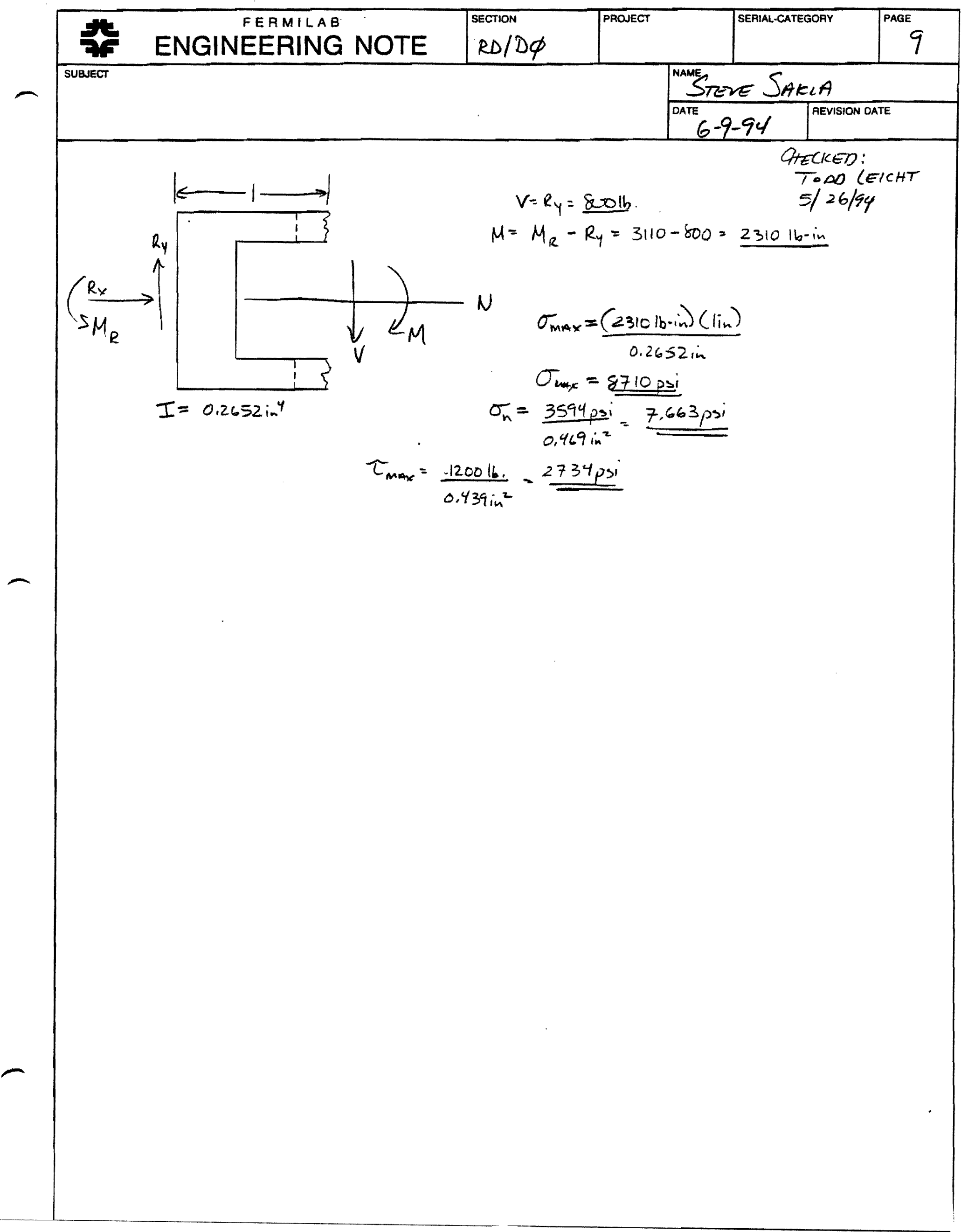




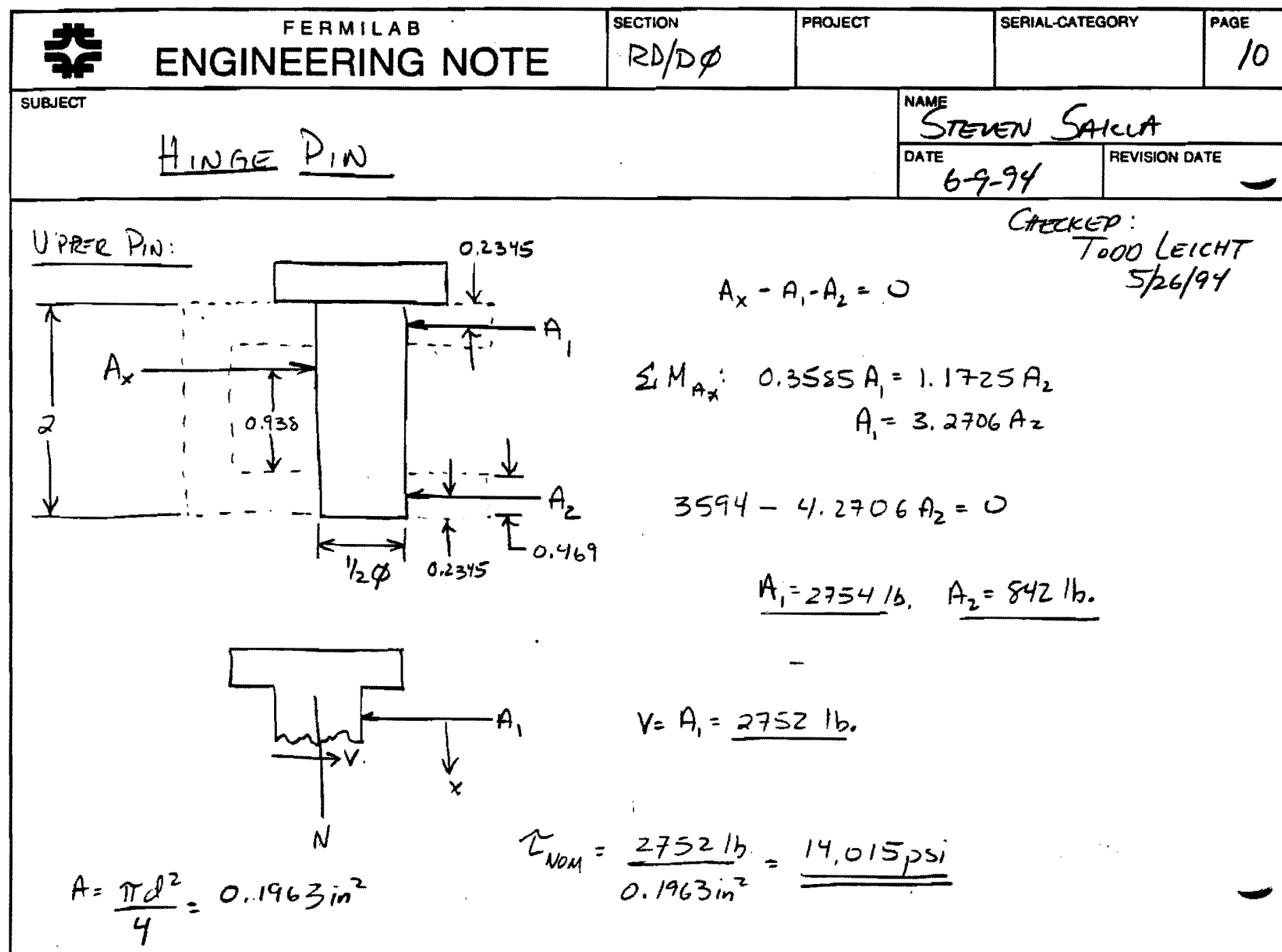

LOWER PIN:

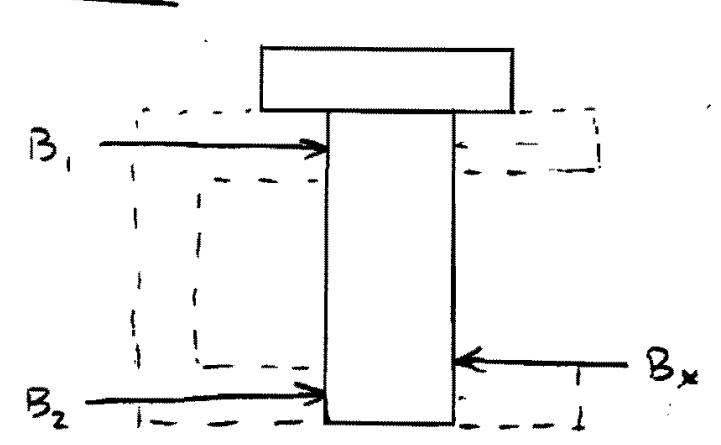

$$
\begin{aligned}
& B_{x}=3594 \mathrm{lb} \\
& \sum H_{B_{x}}=0.2345 B_{2}=1.2965 \mathrm{~B}, \\
& B_{1}+B_{2}=B_{x}=3594.5288 B_{1} \\
& B_{1}=550 \mathrm{lb} . \quad B_{2}=3044 \mathrm{bb} .
\end{aligned}
$$

* Same dimewslows as upper pin

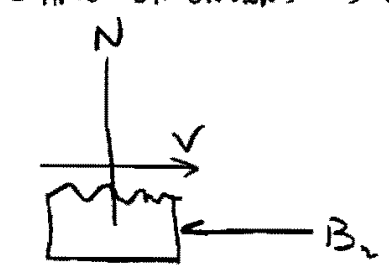

$$
\tau_{\text {Nom }}=\frac{V}{A}=\frac{3044 \cdot 1 \mathrm{~b}}{0.1963 \mathrm{in}^{2}}=15,504 \mathrm{psi}
$$

$$
A=\frac{\pi d^{2}}{4}=0.1963 \mathrm{in}^{2} \quad V=B_{2}
$$




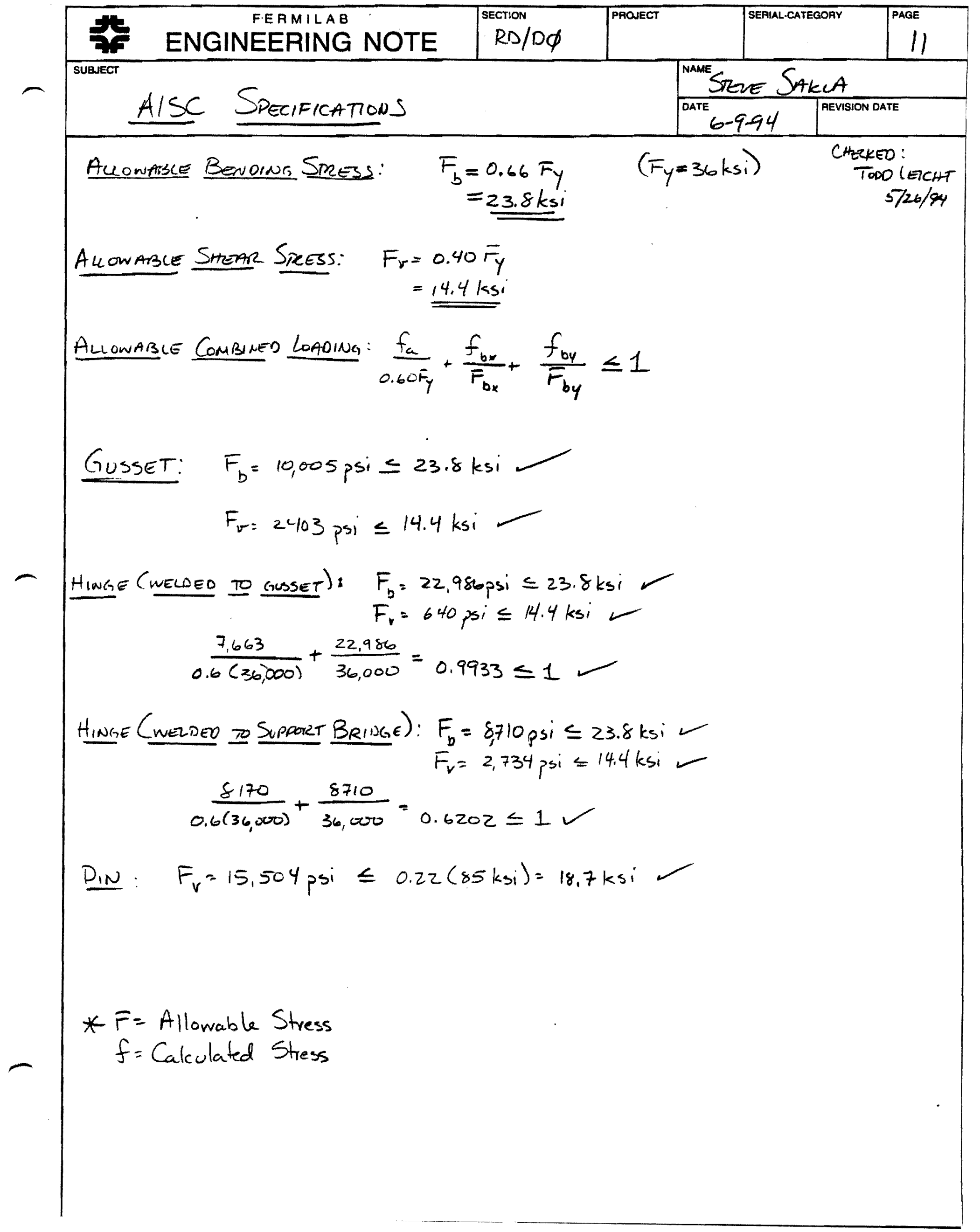




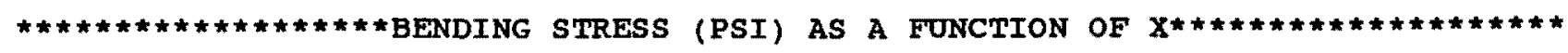

$\begin{array}{ll}x= & 0.5000000 \\ x= & 1.500000 \\ x= & 2.500000 \\ x= & 3.500000 \\ x= & 4.500000 \\ x= & 5.500000 \\ x= & 6.500000 \\ x= & 7.500000 \\ x= & 8.500000 \\ x= & 9.500000 \\ x= & 10.50000 \\ x= & 11.50000 \\ x= & 12.50000 \\ x= & 13.50000 \\ x= & 14.50000 \\ x= & 15.50000 \\ x= & 16.50000 \\ x= & 17.50000 \\ x= & 18.50000 \\ x= & 19.50000 \\ x= & 20.50000 \\ x= & 21.50000 \\ x= & 22.50000 \\ x= & 23.50000 \\ x= & 24.50000 \\ x= & 25.50000 \\ x= & 26.50000 \\ x= & 27.50000 \\ x= & 28.50000 \\ x= & 29.50000 \\ x= & 30.50000 \\ x= & 31.50000 \\ x= & 32.50000 \\ x= & 33.50000 \\ & \end{array}$

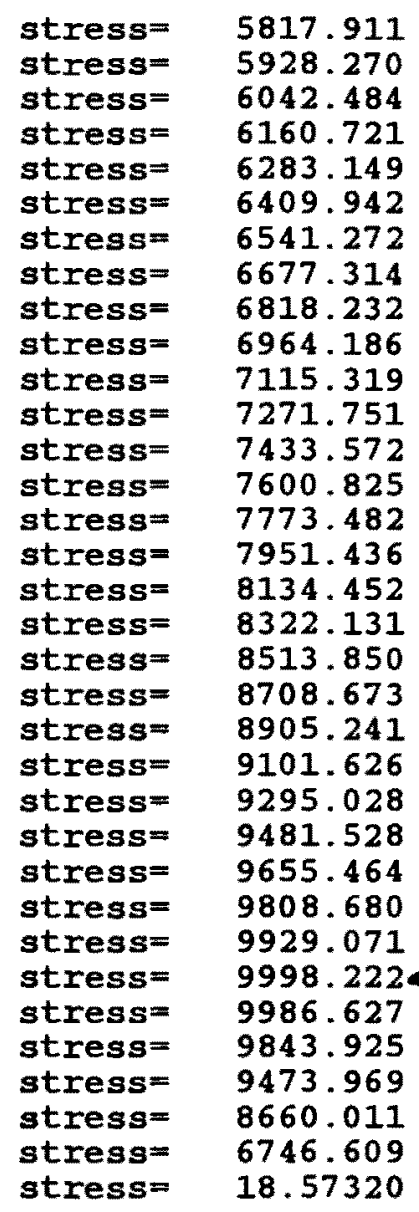




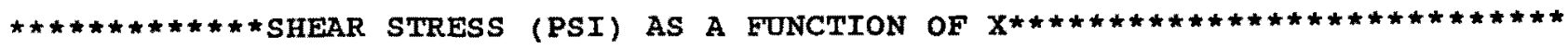

\begin{tabular}{|c|c|}
\hline$x=$ & 0.5000000 \\
\hline$x=$ & 1.500000 \\
\hline$x=$ & 500000 \\
\hline$k=$ & 3.500000 \\
\hline$x=$ & 4.500000 \\
\hline$x=$ & 5.500000 \\
\hline$x=$ & 6.500000 \\
\hline$x=$ & 7.500000 \\
\hline$x=$ & 8.500000 \\
\hline$x=$ & 9.500000 \\
\hline$x=$ & 10.50000 \\
\hline$x=$ & 11.50000 \\
\hline$x=$ & 12.50000 \\
\hline$x=$ & 13.50000 \\
\hline$x=$ & 14.50000 \\
\hline $\mathbf{x}=$ & 15.50000 \\
\hline$x=$ & 16.50000 \\
\hline$x=$ & 17.50000 \\
\hline $\mathbf{x}=$ & 18.50000 \\
\hline $\mathbf{x}=$ & 19.50000 \\
\hline$x=$ & 20.50000 \\
\hline $\mathbf{x}=$ & 21.50000 \\
\hline$x=$ & 22.50000 \\
\hline$x=$ & 23.50000 \\
\hline$x=$ & 24.50000 \\
\hline$x=$ & 25.50000 \\
\hline$x=$ & 26.50000 \\
\hline$x=$ & 27.50000 \\
\hline$x=$ & 28.50000 \\
\hline$x=$ & 29.50000 \\
\hline$x=$ & 30.50000 \\
\hline$x=$ & 31.50000 \\
\hline$x=$ & 32.50000 \\
\hline$x=$ & 50000 \\
\hline
\end{tabular}

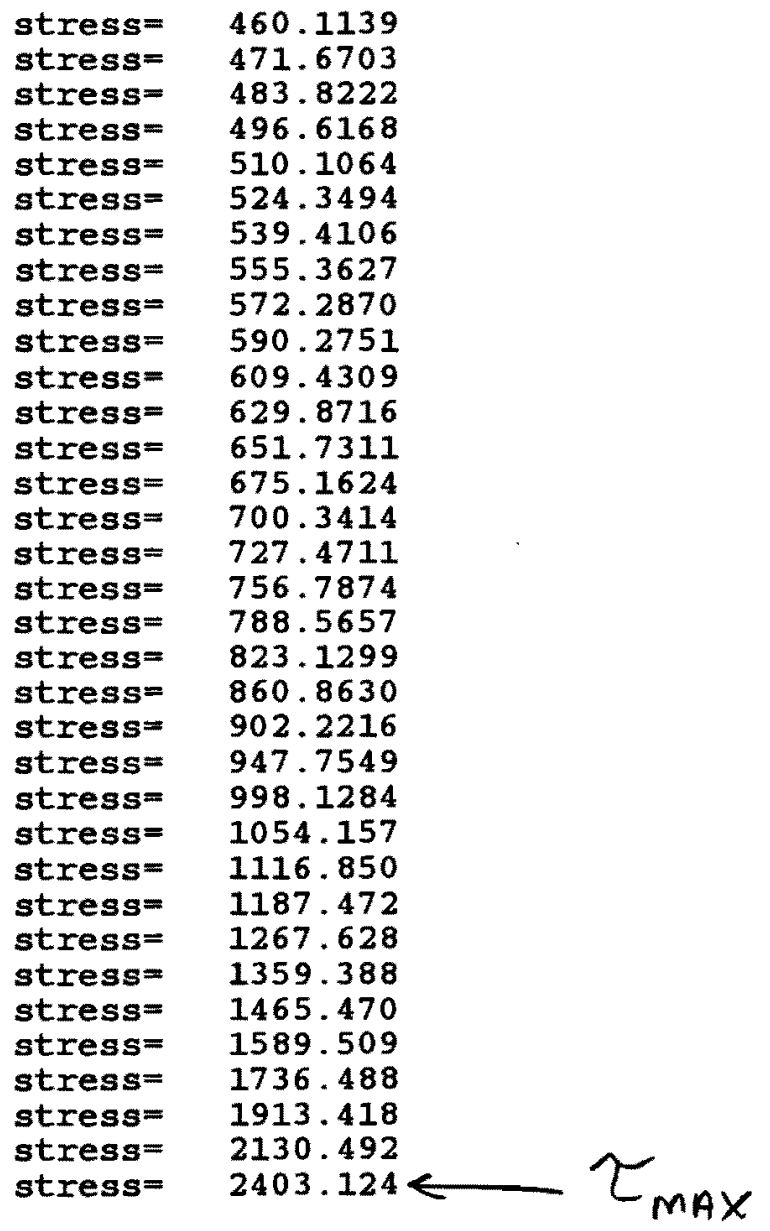

